

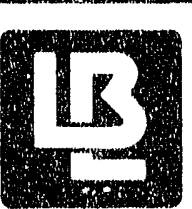

\section{Lawrence Berkeley Laboratory}

UNIVERSITY OF CALIFORNIA

ENERGY \& ENVIRONMENT

DIVISION

To be presented at the 24 tis Internationai Symposium on

Combustion, Sydney, Australia, July 5- 0, 1092,

and to be published in the Proceedings

Flame Front Geometry in Premixed Turbulent Flames

I.G. Shepherd and Wm.T. Ashurst

December 1991

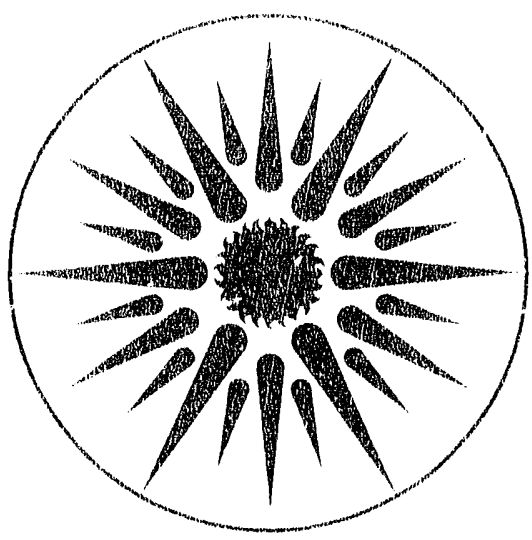

ENERGY \& ENVIRONMENT DIVISION 
DISCLAIMER

This document was prepared as an account of work sponsored by the United States Government. Nolther the United States Government nor any agency thereof, nor The Regents of the University of California, nor any of their employees, makes any warranty, express or implied, or assumes any legal liabillty or responsibility for the accuracy, completeness, or usefulness of any information, apparatus, product, or process disclosed, or represents that its use would not infringe privately owned rights. Reference herein to any specific commercial product, process, or service by its trade name, trademark, manufacturer, or otherwise, does not necessarily constitute or imply its endorsement, tecommendation, or favoring by the United States Covernment or any agency the renf, or The Regents of the University of California. The views and ipinions of authors expressed herein do not necessarily state or reflect those of the United States Government or any agency thereof or The Regents of the University of California and shall not be used for advertising or product endorsement purposes.

Lawrence Berkeley Laboratory is an equal opportunity employer. 


\title{
Flame Front Geometry in Premixed Turbulent Flames
}

\author{
I.G. Shepherd \\ Combustion Group \\ Energy and Environment Division \\ Lawrence Berkeley Laboratory \\ University of California \\ Berkeley, CA 94720 \\ and \\ Wm.T. Ashurst \\ Combustion Research Facility \\ Sandia National Laboratories \\ Livermore, CA 94551-0969
}

December 1991

This work was supported by the Director, Office of Energy Research, Office of Basic Energy Sciences, Chemical Sciences Division, of the U.S. Department of Energy under Contrazt No. DE-AC03-76SF00098. 


\title{
FLAME FRONT GEOMETRY IN PREMIXED TURBULENT FLAMES
}

\author{
I.G. Shepherd and Wm.T. Ashurst* \\ Combustion Group, \\ Energy and Environment Division \\ Lawrence Berkeley Laboratory \\ California 94720 \\ *Combustion Research Facility \\ Sandia National Laboratories \\ Livermor, California 94551-0969
}
Corresponding Author: I.G.Shepherd
B29C Lawrence Berkeley laboratory
1 Cyclotron Rd.
Berkeley, CA 94720
(510) 486-5438
(510) 486-4122 FAX
E-mail:rkc@csa.lbl.gov

Subjects:

(5.0) Experimental Methods

(5.1) Laser Diagnostics

(10.3) Numerical-multidimensional

(14.2) Premixed Turbulent Combustion

Paper Length:

Text:

3250 words

Figures: $\quad 6 \times 200 \quad 1200$ words

Tables: $\quad 2 \times 200 \quad 400$ words

Total : $\quad \mathbf{4 8 5 0}$ words 


\begin{abstract}
Experimental and numerical determinations of flame front curvature and orientation in premixed turbulent flames are presented. The experimental data is obtained from planar, cross sectional images of stagnation point flames at high Damköhler number. A direct numerical simulation of a constant energy flow is combined with a zero-thickness, constant density flame model to provide the numerical results. The computational domain is a $32^{3}$ cube with periodic boundary conditions. The two-dimensional curvature distributions of the experiments and numerical simulations compare well at similar $\mathrm{q}^{\prime} / \mathrm{S}_{\mathrm{L}}$ values with means close to zero and marked negative skewness. At higher turbulence levels the simulations show that the distributions become symmetric about zero. These features are also found in the three dimensional distributions of curvature. The simulations support assumptions which make it possible to determine the mean direction cosines from the experimental data. This leads to a reduction of $12 \%$ in the estimated flame surface area density in the middle of the flame brush.
\end{abstract}

\title{
INTRODUCTION
}

At high Damköhler and Reynolds numbers the flame brush of a premixed turbulent flame can be treated ${ }^{1}$ as a region of reactants and products separated by strained laminar flamelets 2,3 . Under these conditions the mean turbulent reaction rate is determined by the flame surface geometry and local burning rate. In Bray's description ${ }^{3}$ the mean reaction rate at a point in the flame zone is

$$
<w>=\rho_{u}\left(S_{L}\right)^{O} I_{0} \Sigma
$$

where $\rho_{\mathrm{u}}$ is the reactant density and $\left(S_{\mathrm{L}}\right)^{\circ}$ the unstrained laminar burning velocity. $I_{0}$ describes the mean effects of the strain field and flamelet curvature on the local burning velocity by the local flame stretch, $\kappa$

$$
\kappa=\frac{-b}{S_{L}}+h_{1}+h_{2}
$$

where $b=n \cdot e \cdot n$ is the strain rate in the plane of the flame and the principal components of curvature, $h_{1}, h_{2}$ are defined below. In equation $1 \Sigma$ is the mean flamelet surface area to volume ratio for which various modeled transport equation; have been developed 4,5 . A simple expression for $\Sigma$ has also been proposed ${ }^{6}$ which is based on the spatial distribution of flame crossings along a contour of constant mean progress 
variable, $<c>(c=0$ in the reactants and $c=1$ in the products $)$ and is also open to direct experimental investigation:

$$
\Sigma=\mathrm{g} \frac{\langle\mathrm{c}>(1-<\mathrm{c}>)}{<\sigma>L}
$$

where $\langle\sigma\rangle$ is a mean direction cosine of the flame normal relative to the $<$ c $>$ contours, $L$ is the scalar integral length scale along the contour and $g$ a constant of order unity.

The use of a thin laser sheet to produce tomographic sections of flame zones 7,8 is a fruitful means of obtaining experimental data in the laminar flamelet regime. With this technique it is possible to obtain, in a variety of configurations, quantitative estimates of scalar length scales, $L^{8},<\sigma>^{9}$ and the flame surface area by fractal analysis ${ }^{10}$.

In this paper experimental and numerical measurements of local flame front curvature and mean orientation (direction cosine) are presented. The experimental results are obtained from laser sheet images of stagnation-point premixed turbulent flames. These planar cuts provide only an estimate of $h$ or $\sigma$ and so the suitability of the present data to test and validate models such as that outlined above is unclear ${ }^{11}$. The direct experimental determination of either of these quantities in three dimensions presents serious practical difficulties. By comparison, however, with three dimensional direct numerical simulations of premixed turbulent flames it will be shown that useful information can be derived from the experimental data.

\section{Numerical Model:}

The investigation of flame propagation in a statistically steady state is possible by the direct numerical simulation of a constant energy turbulent flow. Constant energy is achieved by a large-scale forcing scheme so that the turbulent kinetic energy is fixed at $u^{2}+v^{2}+w^{\prime 2}=3$; the average rms velocity, $\mathrm{q}^{\prime}$, is therefore unity. The computational domain is a periodic cube with an edge length of $L$ and mesh spacing of $L / 32$ giving a total of $32^{3}$ grid points. The measured Taylor length scale is $\sim 0.13 L$ which is of the same order as the largest possible size of $L / 2$ which arises from the periodic boundary conditions. The Taylor Reynolds number is $\sim 60$ and the kinematic viscosity is 0.002 in units where $L=l$ and $q^{\prime}=1$.

A zero-thickness, constant-density premixed flame model described in Kerstein et $a l .{ }^{12}$ has been used in these simulations. A continuous scalar $G$ is convected by the flow and flame propagation is accomplished by Huygens' mechanism, 


$$
\frac{\partial \mathrm{G}}{\partial \mathrm{t}}+\mathbf{u} \cdot \nabla \mathrm{G}=\mathrm{S}_{\mathrm{L}}|\nabla \mathrm{G}|
$$

where the flame speed $S_{L}$ is a function of strain and curvature and $\mathbf{u}$ is the turbulent flow field. The flame speed is $S_{L}=\left(S_{L}\right)^{\circ} \exp \left(-L_{M} \kappa\right)$ where $L_{M}=L / 40$ is a Markstein length representing the effects of the local flame stretch, $\kappa$, on the local burning velocity. An exponential form for the burning velocity is used so that large stretch can only reduce the flame speed to zero and an upper bound is imposed so that $-L_{M} \mathrm{~K} \leq 1.39$ i.e. $\left(S_{L} /\left(S_{L}\right)^{0}\right)_{\text {max }}=4$. With respect to any value of the scalar, $G$, the unburnt fluid is located at smaller values of $G$. In this constant density flame simulation, every computational grid point contributes statistical information about the flame surface geometry which is described by the unit normal vector $n=-\nabla G /|\nabla G|$ and the two principal curvatures of the surface $h_{1}=1 / R_{1}$ and $h_{2}=1 / R_{2}$. At each grid point the first and second spatial derivatives of $G$ are calculated and the elements of the curvature tensor are formed

$$
h_{i j}=-\frac{\partial^{2} G}{\partial x_{i} \partial x_{j}} \frac{1}{d}+\frac{1}{d^{3}} \sum \frac{\partial G}{\partial x_{i}} \frac{\partial G}{\partial x_{k}} \frac{\partial^{2} G}{\partial x_{j} \partial x_{k}}
$$

where $d=|\nabla G|$. This tensor has two real eigenvalues $h_{1}, h_{2}$ and one eigenvalue equal to zero (positive curvature is convex to the reactants).

\section{EXPERIMENTAL DETAILS}

A tomographic study was performed on methane/air and ethylene/air stagnationpoint, premixed turbulent flames where the Damköhler number derived from the integral time scale of the reactant stream, the chemical reaction time based on the kinematic viscosity of the reactants and the laminar burning velocity is large $(\sim 80)$ indicating that the flames are in the wrinkled laminar flame regimel. Scalar properties, such as temperature or density, can be determined by the measurement of the intensity of light scattered from micron sized oil droplets which are seeded in the reactant stream and evaporate at the flame front. The oil droplets are illuminated by a laser sheet and photographed in the direction normal to the sheet.

An axisymmetric flow of premixed fuel and air at an exit velocity of $5 \mathrm{~m} / \mathrm{s}$ is provided by a $50 \mathrm{~mm}$ diameter nozzle. A co-flowing air stream at the same velocity shields the inner flow from interaction with the room air. The reactant flow turbulence $(7 \%)$, generated by a perforated plate placed $50 \mathrm{~mm}$ upstream of the burner nozzle, has an integral length scale of $3 \mathrm{~mm}$ and the turbulent Reynolds number based on these values is 
70. A stagnation plate is positioned $100 \mathrm{~mm}$ downstream of the nozzle exit. The burner configuration has been described in detail elsewhere ${ }^{8}$. The flow geometry chosen for this study has significant advantages in that the tomographic cross sections through the stagnation line provide a good statistical representation of the scalar field due to the axisymmetry of the system.

The light source is a copper vapor laser which delivers $5 \mathrm{~mJ}$ pulses with a 20$30 \mathrm{nsecs}$ pulse width at a repetition rates of $4 \mathrm{kHz}$ and hence it is possible to resolve the instantanecus flame shape. The $38 \mathrm{~mm}$ diameter laser beam is transformed by cylindrical lenses to a sheet $0.6 \mathrm{~mm}$ thick by $50 \mathrm{~mm}$ high with a field of view of $60 \mathrm{~mm}$. The reactant flow is seeded with silicone oil droplets $(\sim 1 \mu \mathrm{m}$ in diameter) which evaporate at the flame front $(\sim 500 \mathrm{~K})$. The laser sheet is recorded by a high speed $16 \mathrm{~mm}$ Fastax camera which provides the trigger pulse for the laser.

The film is digitized to give 512 by 512 pixel images with 256 gray scales of light intensity resulting in horizontal and vertical resolutions of 0.155 and $0.121 \mathrm{~mm} / \mathrm{pixel}$, respectively. In a digitized image the flame boundary is clearly delineated as the interface between the light (cold reactants with seed particles) and dark (hot products without particles) regions of the image. Flame boundaries defined by an intensity threshold, are generated by an edge finding algorithm which produces a continuous flame edge. The threshold is determined by inspection of the histogram of pixel intensity: the results are not sensitive to the precise value of the threshold.

\section{RESULTS AND DISCUSSION}

Flame Front Curvature:

The experimental data consists of a set of two hundred flame front boundaries for each case, sse Table I, which are derived from the tomographic record in the manner descrihed above. Care was taken to ensure that they represented a good statistical sample by widely spacing the film frames which were analyzed. The flame boundaries were smonthed to remove digitization noise ${ }^{13}$ and, for a window of $35 \mathrm{~mm}$ around the stagnation line the local curvature along the boundary was obtained at each point by the least mean square fit of a circle. The curvature distribution, derived form forty images, was not very sensitive to the number of points in the fit and nine points, centered on the point to be fitted, were used in all cases. This procedure results in a minimum resolvable curvature of $\pm 5 \mathrm{~mm}^{-1}$.

The probability distributions ( $\mathrm{df}$ ) of curvature normalized by the standard deviation of the distribution for all the cases are shown in figure 1. In Table I the mean 
and standard deviations of the experimental results have units of $\mathrm{mm}^{-1}$ and for $\mathrm{N} 1$ and $\mathrm{N} 2$ the units are the Taylor microscale. The areas under all the pdfs are normalized to unity. The mean curvatures are close to zero but all the distributions are negatively skewed. This may be explained by the importance of Huygens propagation of the flame front under the moderate turbulence conditions $\left(q^{\prime} / S_{L} \sim 0.6\right.$ at the cold boundary of the flame) when cusps are formed as regions of negative curvature are consumed faster than the outward-propagating convex portions of the flame front. If the flame response to curvature is linear ${ }^{14}$ and, as found here, $\langle h\rangle=0$ then $I_{0}$ in equation 1 can be modeled ${ }^{15}$ solely from a knowledge of the distribution of the strain rates and a 'library' of strained laminar flame calculations.

Table I

Flame Front Curvature

\begin{tabular}{|c|c|c|c|c|c|c|c|}
\hline case & fuel & $\phi$ & $\langle\mathrm{h}\rangle$ & $\mathrm{h}^{\prime}$ & skewness & kurtosis & $\mathrm{q}^{\prime} / \mathrm{S}_{\mathrm{L}}$ \\
\hline $\mathrm{S} 1$ & methane & 1.0 & 0.0048 & 0.61 & -1.19 & 8.2 & 0.68 \\
\hline $\mathrm{S} 9$ & ethylene & 1.0 & -0.0048 & 0.76 & -1.10 & 8.5 & 0.56 \\
\hline $\mathrm{S} 10$ & ethylene & 0.85 & -0.0026 & 0.62 & -1.88 & 9.84 & 0.62 \\
\hline $\mathrm{S} 11$ & ethylene & 0.75 & 0.0025 & 0.54 & -1.71 & 10.3 & 0.64 \\
\hline $\mathrm{N} 1$ & - & - & 0.0051 & 1.39 & -1.31 & 8.00 & 0.50 \\
\hline $\mathrm{N} 1_{3 \mathrm{D}}$ & - & - & 0.0029 & 1.81 & -1.19 & 5.85 & 0.50 \\
\hline $\mathrm{N} 2$ & - & - & -0.0156 & 3.38 & 0.15 & 13.00 & 4.00 \\
\hline $\mathrm{N} 2_{3 D}$ & - & - & 0.0010 & 3.19 & -0.14 & 7.35 & 4.00 \\
\hline
\end{tabular}

The experimental results are also compared in figure 1, to curvature distributions obtained from the numerical simulations where the curvatures are evaluated on planes parallel to the propagation direction. The numerical simulation N1 (solid line) compares well with the experimental results which are at a similar $\mathrm{q}^{\prime} / \mathrm{S}_{\mathrm{L}}$ value. A marked change in the distribution occurs, however, when $q^{1} / S_{L}$ is increased. In the case $N 2$, where $q^{1} / S_{T}=$ 4 , a symmetric distribution (dashed line) of curvature is observed because turbulent fluid motion dominates flame propagation in determining flame shape.

The numerical curvature distribution in two dimensions (2D) is compared to that in three dimensions (3D) in Figure 2. The 3D curvature at a point on the surface, $h_{3 D}$, is defined as the sum of the principal curvatures: $h_{3 D}=h_{1}+h_{2}$. The 2 ar $13 D$ curvatures are calculated at each grid point, sorted by the $2 \mathrm{D}$ curvature and then binned to give 
equal numbers of samples in each bin. The mean 2D and 3D curvatures in each bin are presented in figure 2, the dashed line has a slope of unity. Except at cu vatures far from zero where the statistical uncertainty is high the curvature distributions are similar indicating that the experimental results are a reasonably good estimate of the curvature of the surface. Furthermore, the shape of the pdfs of $2 \mathrm{D}$ curvature are comparable to those of $h_{3 D}$, figure 3 and table I, having similgr means and skewness factors. It is of interest that the distributions of principal curvature obtained by Rutland et.al. 16 from direct numerical simulations with a $128^{3}$ computational domain, one-step chemistry and decaying turbulence, are symmetrical about zero and also independent of $\mathrm{q}^{\prime} / \mathrm{S}_{\mathrm{L}}$. Analysis of the same database ${ }^{17}$ in terms of $h_{3 D}$, however, revealed similar distributions to those found here.

The local flame geometry can be further elucidated by considering the shape factor, $S_{h}$, which is the ratio of the principal curvatures: the smaller curvature (in magnitude) being divided by the larger to yiold a result bounded by $\pm 1: S_{h}=h_{1} / h_{2}$. A spherical shape has equal curvatures, a zero value is that of a cylinder (one curvature is zero), and a saddle point has curvatures of equal magnitude but opposite sign. Pope et al 18 have calculated and displayed the curvature ratio of material elements in this manner. Figure 4 presents the distribution of flame curvature shape for N1 and N2. The flame results agree with the behavior of material points in having a vanishing probability for spherical shapes and a most probable shape to be that of a cylinder. The shape factor also does not change significantly with $\mathrm{q}^{\prime} / \mathrm{S}_{\mathrm{L}}$ althe'igh the distribution of $h_{3 D}$ is varies markedly with $q^{\prime} / S_{L}$. The cylindrical behavior is a result of the intense vorticity being tube-like (see the cover of Physics Today, January 19S0). As a flame approaches such a structure, it becomes wrapped around the vortex, and so the flame surface is more often cylindrical than spherical in shape. A saddle shape will be created by those vortex tubes which are bent. A stretching vortex has a large strain rate in an annular region outside the vortex core thus flame regions with large curvature will occur outside the vortical core where the shearing strain is largest. In deciding on the use of a single parameter to represent the curvature it may be noted that $h_{3 D}$ will be close tu zero not only when $h_{1}$ $\ll h_{2}$ but also at saddle points where $h_{1}$ and $h_{2}$ are equal in magnitude but of oppositc sign.

\section{Direction Cosines:}


To clarify the presentation of the data for flame front orientation reference will be made to the coordinate system shown in figure 5 . The direction of mean propagation of the flame is along the $x$ axis and $y z$ is a plane of $\langle c\rangle$. The direction cosine in equation 3 , $\sigma_{\theta}$, is the cosine of the angle $(\theta)$ formed between the instantaneous flame front normal (the bold line) and the yz plane. The laser sheet is represented by the xy (or equivalent $\mathrm{xz}$ ) plane and the direction cosine determined by experiment is therefore formed by the projection of $\theta$ on the xy plane $\left(\phi_{y}\right)$. The angle between the $y$ axis and the projection of the flame front normal on the yz plane is $\alpha$. By simple geometrical argument it can be shown that:

$$
\tan (\theta)=\tan (\phi) \cos (\alpha)
$$

The direction cosines, $\sigma_{\phi}$, were determined experimentally by superimposing progress variable contours, obtained by averaging all the images ${ }^{8}$, on 200 individual flame front boundaries and calculating the angle between the contour and flame edge at each intersection point. The results are presented in table II. The mean direction cosine averaged over all the cases at $\langle c\rangle=0.5$ is 0.651 and the mean angle is $45^{\circ}$. Chew et al. ${ }^{9}$ found in similar planar measurements in Bunsen flames that $\sigma \sim 0.5$ and that it did not vary with $\langle c\rangle$. The present results, however, show a clear trend of $\left\langle\sigma_{\phi}\right\rangle$ increasing with $<c\rangle$. This trend is consistent with the curvature results which show the flame fronts to be cusped towards the burned gases.

Table II

Direction Cosines

\begin{tabular}{|c|c|c|c|c|c|}
\hline case & $\langle c\rangle$ & $\left\langle\sigma_{\phi}\right\rangle$ & $\left\langle\sigma_{\theta}\right\rangle$ & $\langle\phi\rangle$ & $\langle\theta\rangle$ \\
\hline \hline$S 1$ & 0.25 & 0.565 & 0.643 & 52.3 & 44.6 \\
\hline$S 1$ & 0.50 & 0.631 & 0.696 & 47.3 & 40.2 \\
\hline$S 1$ & 0.75 & 0.671 & 0.742 & 43.0 & 35.3 \\
\hline$S 9$ & 0.25 & 0.623 & 0.703 & 47.5 & 39.3 \\
\hline$S 9$ & 0.50 & 0.686 & 0.752 & 42.1 & 34.5 \\
\hline$S 9$ & 0.75 & 0.728 & 0.786 & 37.7 & 30.8 \\
\hline$S 10$ & 0.50 & 0.639 & 0.706 & 46.5 & 39.2 \\
\hline$S 11$ & 0.50 & 0.646 & 0.721 & 46.1 & 37.9 \\
\hline
\end{tabular}

Equation 6 shows that the experimental results will overestimate the true direction cosine, $\sigma_{\theta}$. If, however, $\phi$ and $\alpha$ are statistically independent then, from a knowledge of 
the distribution of $\alpha, \sigma_{\theta}$ can be calculated from the experimental data. The flow is axisymmetric around the stagnation line and this implies that rotation around the $\mathrm{x}$ axis in figure 5 will not change the pdf of $\phi$ i.e. $P\left(\phi_{y}\right)=P\left(\phi_{z}\right)$ and that $P(\alpha)$ would therefore have the same probability at all values. These assumptions are investigated by an examination of the results of the numerical simulations.

In the numerical simulations the analogue of the intersection point of an instantaneous flame with the $\langle c\rangle=0.5$ contour is defined as when the local value of $G$ equals the $\langle\mathrm{G}\rangle$ of a selected yz plane. For case $\mathrm{N} 1$ it was found that the pdfs $\mathrm{P}\left(\phi_{\mathrm{y}}\right)$ and $\mathrm{P}\left(\phi_{\mathrm{z}}\right)$ were not identical probably due to the persistence of large structures in the computational domain over the ratl.er limited averaging period, $\sim 6 \lambda / q^{\prime}$. When, however, $P\left(\phi_{z}\right)$ is conditioned on the value of $\phi_{y}$ statistical independence is observed, see inset in figure 6. This result supports the assumptions outlined above and $\sigma_{\theta}$ can be calculated from the experimental results assuming $\alpha$ to have equal probability at all angles.

Values were selected randomly ${ }^{8}$ from the experimental pdf of $\phi$ and the pdf of $\alpha$ and a pdf of $\sigma_{\theta}$ was generated by the use of equation 6 . The results are presented in figure 6 for S1 and mean values for all cases are given in Table II. The mean direction cosine at $\langle\mathrm{c}\rangle=0.5$ has become 0.707 and the mean angle $38^{\circ}$. This change represents a $12 \%$ decrease in the value of $\Sigma$ that would be determined in equation 3 from $\left\langle\sigma_{\phi}\right\rangle$.

\section{CONCLUSIONS}

In this paper a combination of experimental and numerical approaches has been exploited to investigate aspects of flame front geometry which are important to current models of premixed turbulent flames in the flamelet regime. Experimental data of curvo ure and direction cosines obtained from planar cuts through the tlame zone have been interpreted in the light of the results of three dimensional direct numerical simulations.

2D experimental and numerical distributions of curvature compare well at similar $\mathrm{q}^{\prime} / \mathrm{S}_{\mathrm{L}}$ values with significant negative skewness about means of zero. These features are also found in the $3 \mathrm{D}$ numerical simulations which also show that, at higher $\mathrm{q}^{\prime} / \mathrm{S}_{\mathrm{L}}$ where models tend to be most successful, this distribution becomes symmetric.

The simulations also support physically reasonable assumptions which make possible the determination of the true mean direction cosine from the experimental pdf of 
its planar value. This leads to a reduction of $\sim 12 \%$ in the estimated area density, $\Sigma$, compared to the experimental results.

It is encouraging for continuing comparisons of the present type that constant density computations are successful in simulating significant features of the geometry of experimental flames. 


\section{Acknowledgements}

IGS would like to thank Dr. Robert Cheng for assistance with the experiments, Mr. Gary Hubbard for data reduction software and Dr. Larry Kostiuk for many simulating discussions. Work supported by United States Department of Energy, through the Office of Basic Energy Sciences, Division of Chemical Sciences (LBL contract No. DE-AC-0376SF00098).

\section{REFERENCES}

1) Borghi, R., in Recent Advances in Aeronautical Sciences, C.Bruno, C. Casci (Eds), Plenum, N.Y., (1984).

2) Peters, N., 21st Symposium (Int'l) on Combustion, 1231-1250, (1986).

3) Bray, K.N.C. Springer Series in Chemical Physics, 47, 356-375, (1987)

4) Franke, Ch. and Peters, N., Proc. SFB 224, RWTH Aachen, 126-147, (1988).

5) Darabiha, N., Giovangigli, V, Trouvé, A, Candel, S.M., Esposito, E., Lecture Notes in Engineering, 40, 591-637, Springer Verlag, (1989)

6) Bray, K.N.C., Libby, P. and Moss J.B., Comb. Sci. and Tech., 41, 143-172, (1984).

7) Goix P.J., Shepherd, I.G. and Trinité, M., Comb. Sci. and Tech., 63, 273-287, (1989).

8) Shepherd, I.G., Cheng, R.K., and Goix, P.J., 23rd. Symposium (Intnl) on Combustion, 781-787, (1991)

9) Chew, T.C., Bray, K.N.C. and Britter, R., Comb. and Flame, 80, 65-82, (1990)

10) Gouldin, F. C., .Comb. and Flame, 68, 249-266 (1987).

11) Kostiuk, L.W., Ph.D. Thesis, University of Cambridge, (1991).

12) Kerstein, A. R., Ashurst, Wm. T. \& Williams, F. A. 1988 Phys. Rev. A 37, 2728.

13) Shepherd, I.G., Cheng, R.K. and Talbot, L., Exp. in Fluids, to be published (1992).

14) Clavin,P., Prog. in Eng. and Comb. Sci.,11,1-60, (1985).

15) Cant, R.S., and Bray, K.N.C. 22nd Symposium (Int'l) on Combustion, 791-799, (1988). 
16) Rutland, C.J., Ferzinger, J.H. and El Tahry, S.H., 23rd Symposium (Int'l) on Combustion, 621-627, (1990)

17) Cant R.S., Rutland C.J. and Trouvé, A., Proceedings of the Summer Program, Stanford University, (1990).

18) Pope, S. B., Yeung, P. K. \& Girimaji, S. S. Phys, Fluids A, 1(12), 2010, (1989).

\section{FIGURE CAPTIONS}

Figure 1: Comparison of experimental and numerical distributions of two- dimensional curvature normalized by their standard deviations.

Figure 2: Numerical three-dimensional curvature conditioned on the planar values.

Figure 3: Comparison of three dimensional curvatures at $\mathrm{q} \backslash \mathrm{SL}=0.5$ (solid line) and $\mathrm{q}^{\prime} / \mathrm{SL}=4.0$ (dashed line).

Figure 4: Shape factor from simulations.

Figure 5: Co-ordinate system showing flame normal and direction cosines.

Figure 6: Experimental probability distributions $\sigma_{\phi}(2 \mathrm{D})$ and $\sigma_{\theta}(3 \mathrm{D})$. Insei of numerical results of $\phi_{z}$ conditioned on the value of $\phi_{y}$. 


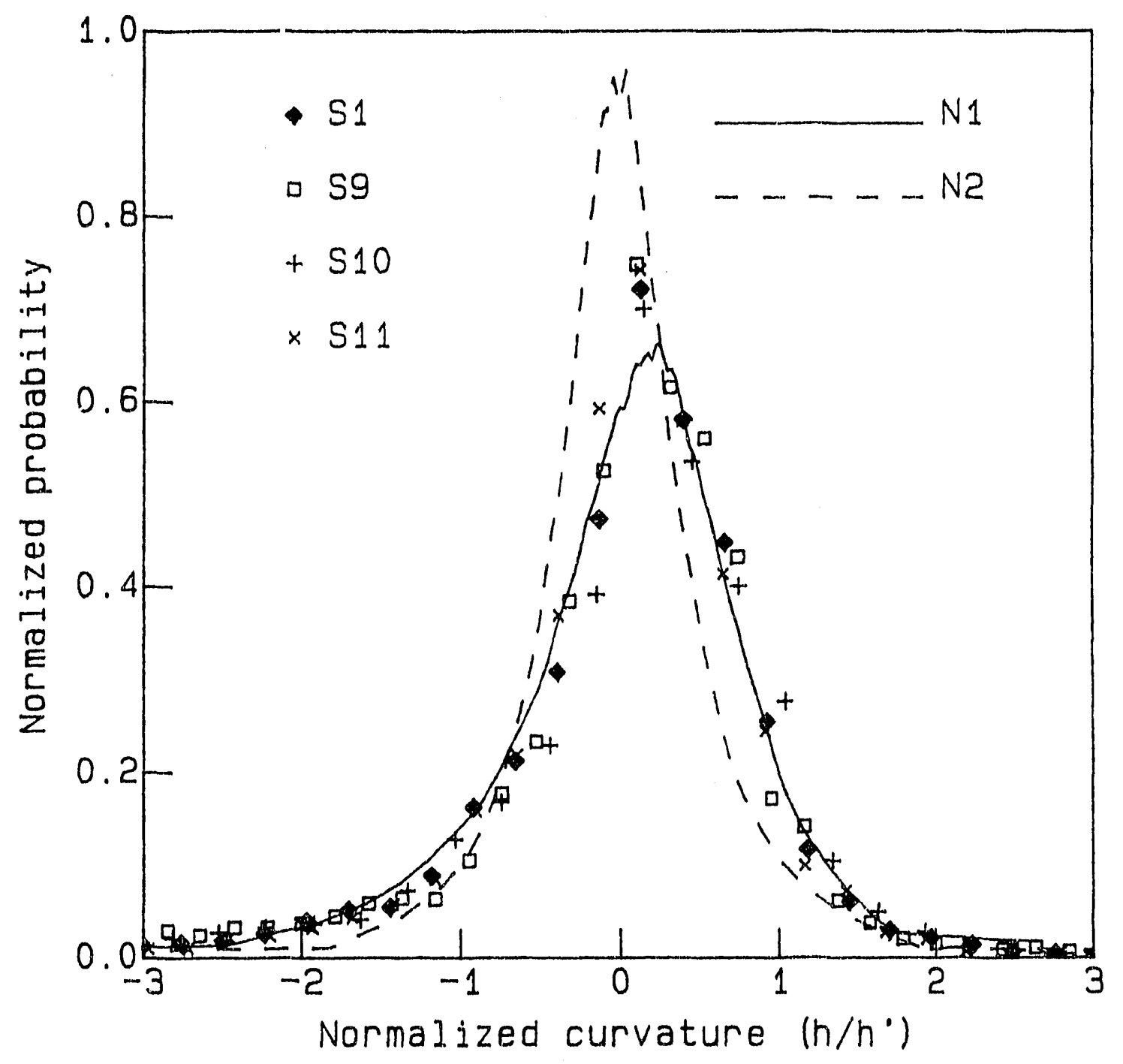

Figure 1 


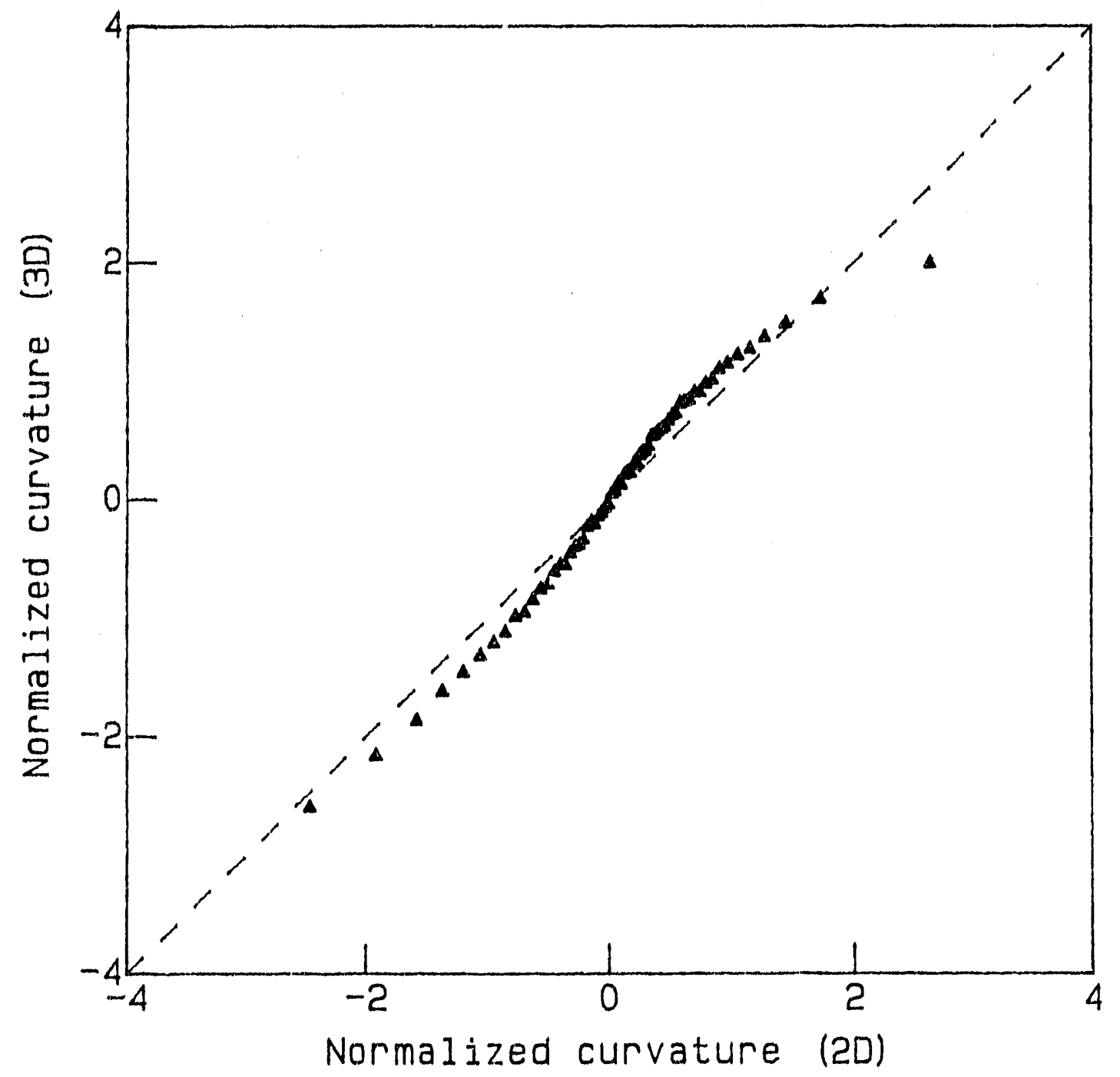

Figure 2 


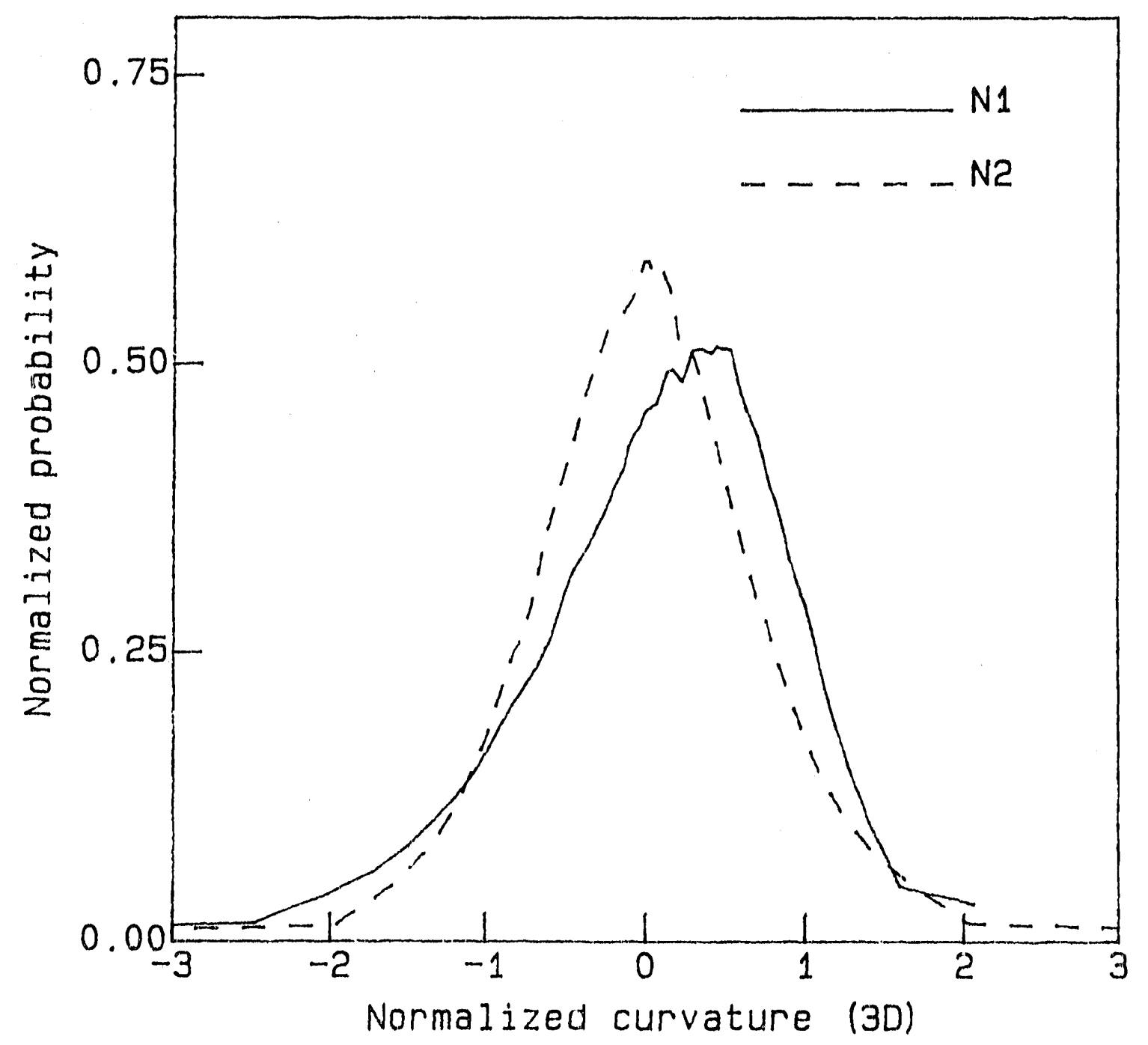

Figure 3 


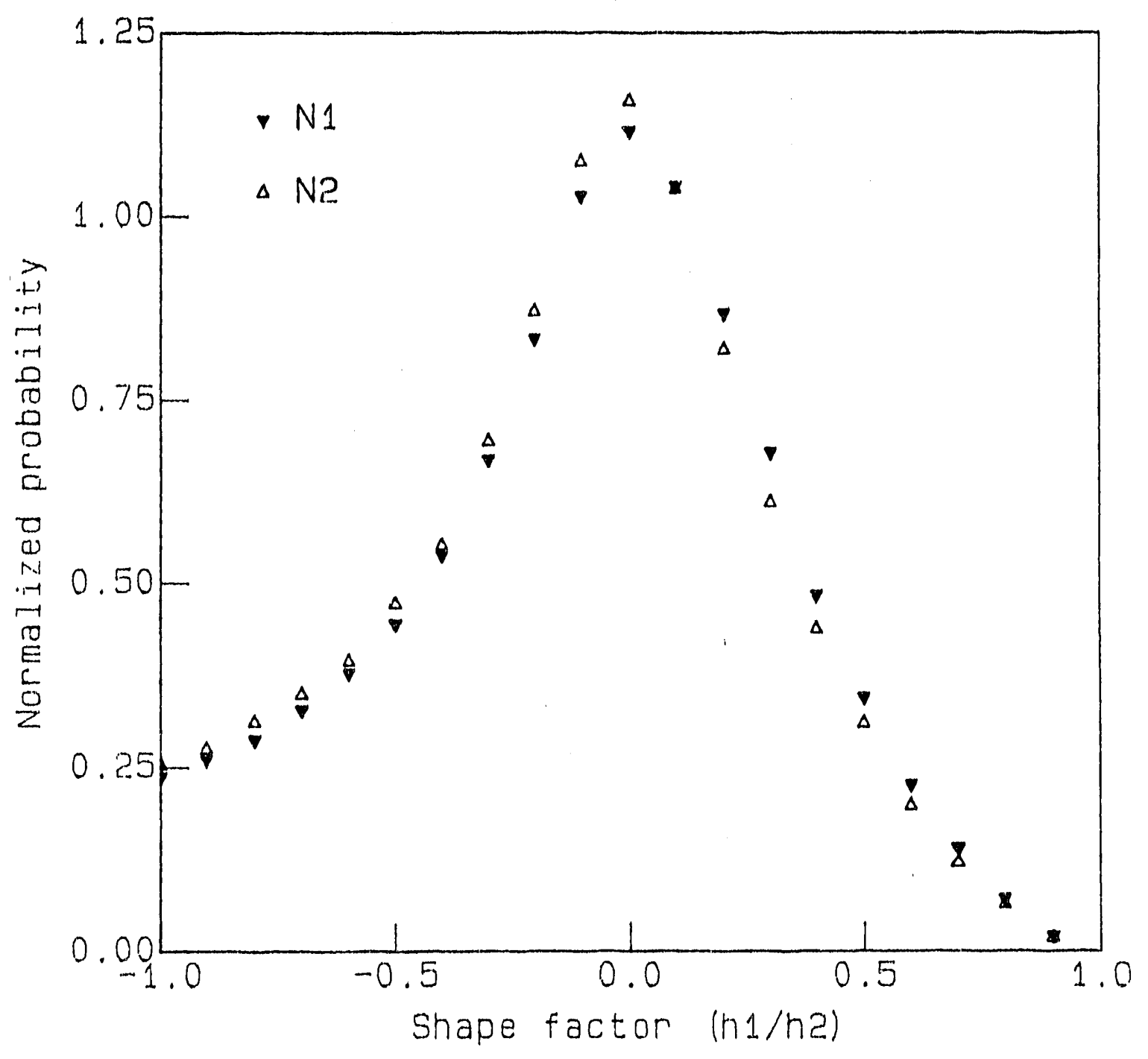

Figure 4 


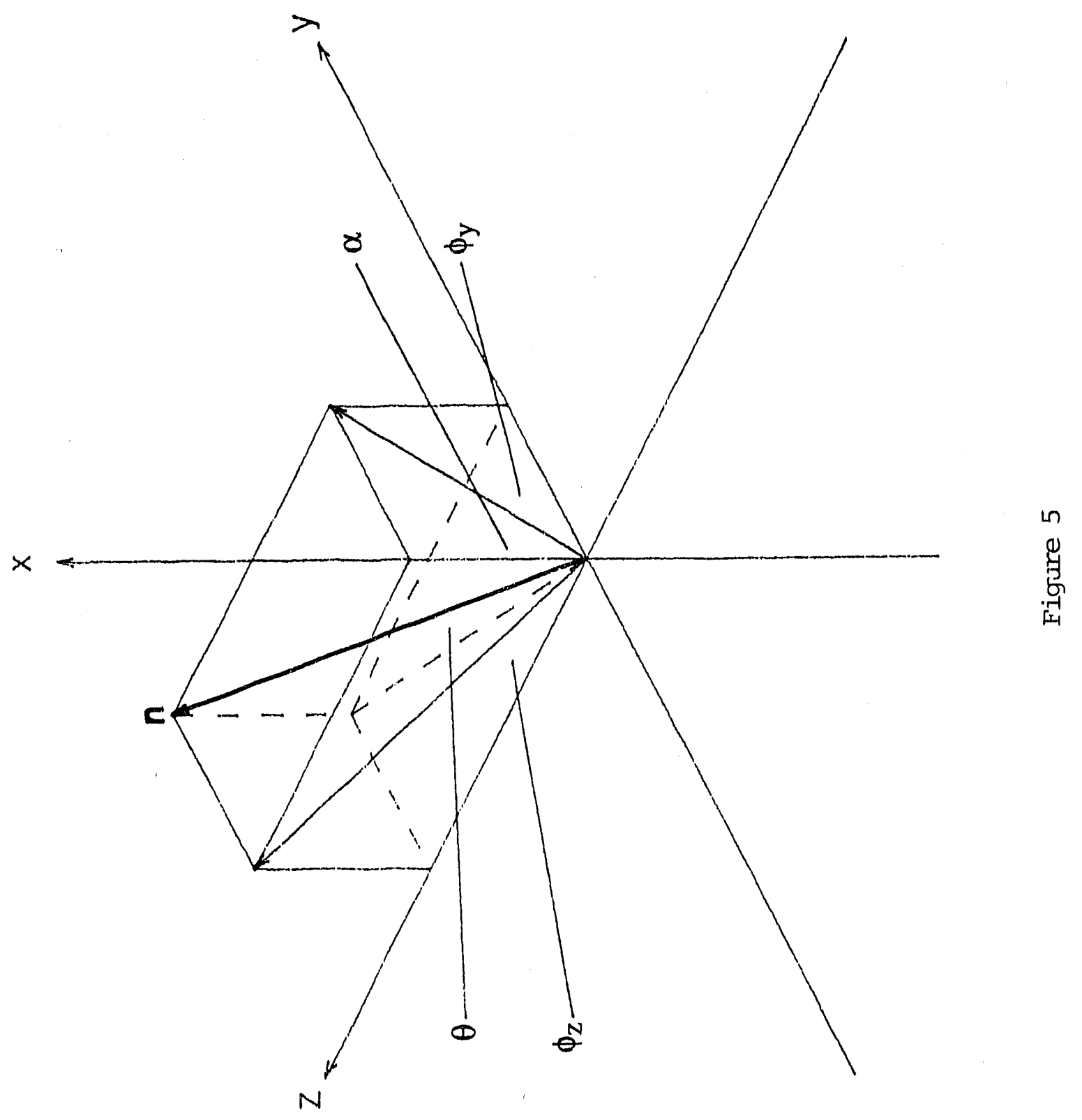




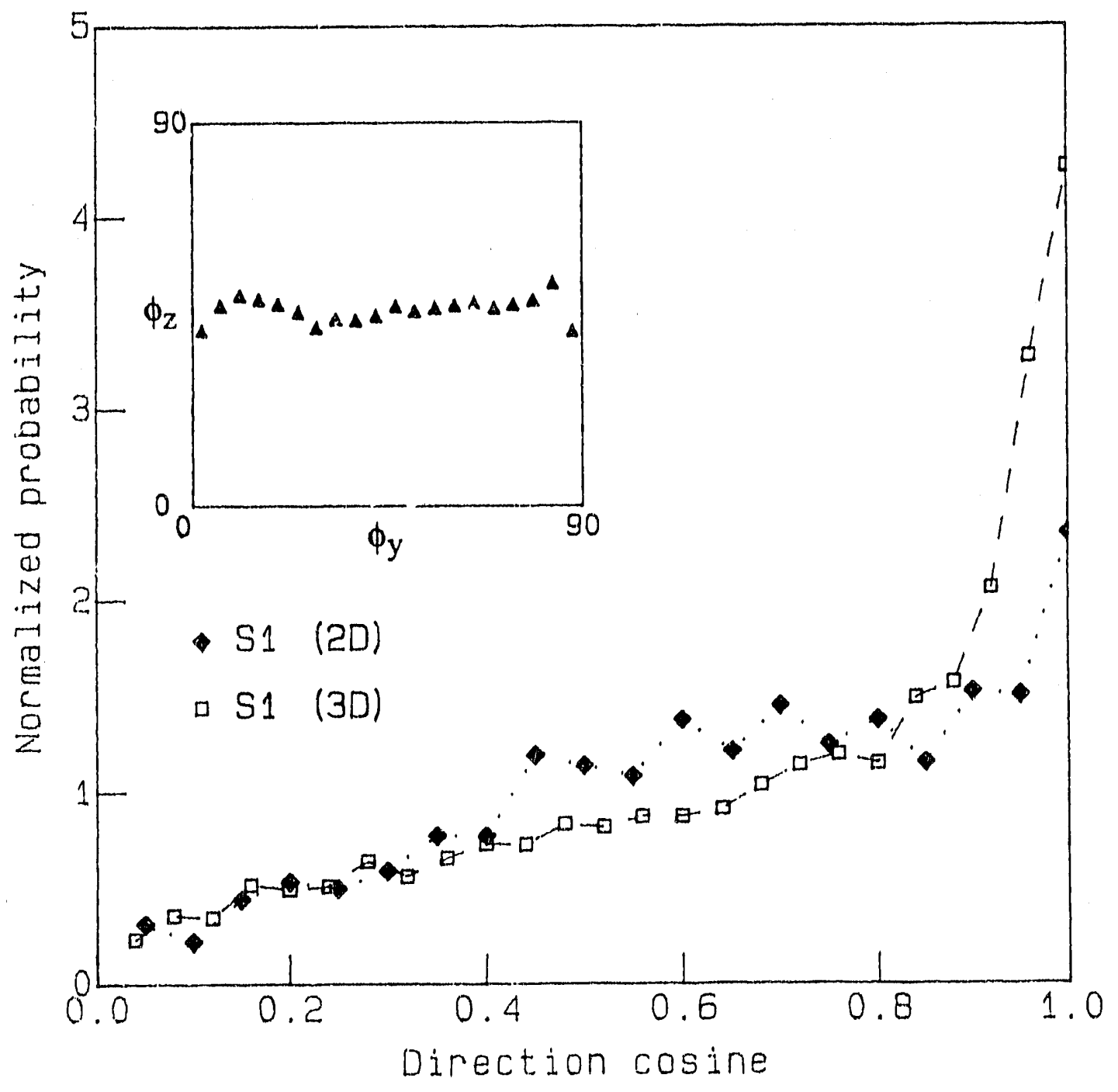

Figure 6 

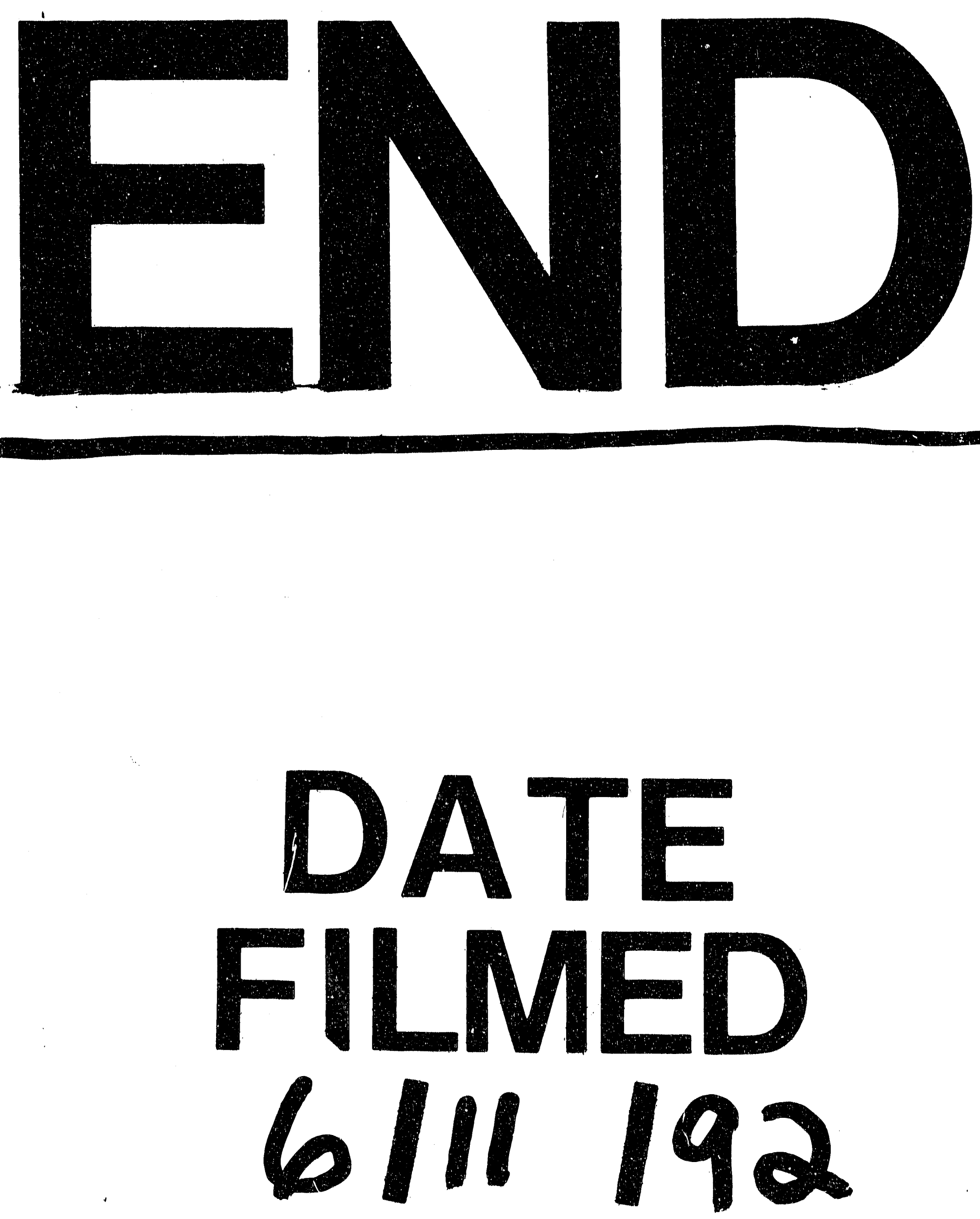


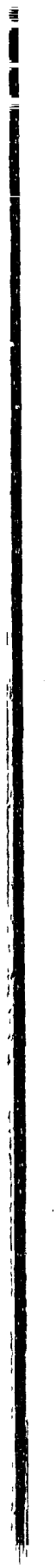

